
$\angle$ Research Square
Preprints are preliminary reports that have not undergone peer review.
They should not be considered conclusive, used to inform clinical practice, or referenced by the media as validated information.

\title{
Effect of Changes in the Posterior Tibial Slope on Femorotibial Articular Contact Kinematics after Cruciate-Retaining Total Knee Arthroplasty: Do the Changes Affect Outcomes?
}

\author{
xiqing pan \\ Third Hospital of Shijiazhuang, 15 Tiyu South Street, Shijiazhuang \\ feng li ( $\sim$ lifengprofessor@126.com ) \\ Third Hospital of Shijiazhuang, 15 Tiyu South Street, Shijiazhuang \\ jinhui liu \\ Third Hospital of Shijiazhuang, 15 Tiyu South Street, Shijiazhuang \\ jiangli zhang \\ Third Hospital of Shijiazhuang, 15 Tiyu South Street, Shijiazhuang \\ an chai \\ Third Hospital of Shijiazhuang, 15 Tiyu South Street, Shijiazhuang \\ lei shu \\ Third Hospital of Shijiazhuang, 15 Tiyu South Street, Shijiazhuang \\ yao li \\ Third Hospital of Shijiazhuang, 15 Tiyu South Street, Shijiazhuang
}

Research Article

Keywords: Tibial, Kinematics, Knee, Arthroplasty, Slope

Posted Date: November 1st, 2021

DOI: https://doi.org/10.21203/rs.3.rs-998813/v1

License: (c) (1) This work is licensed under a Creative Commons Attribution 4.0 International License. Read Full License 


\section{Abstract \\ Background}

Total knee arthroplasty (TKA) outcomes are affected by many factors. This study aimed to evaluate whether changes in the posterior tibial slope (PTS) affects patients' outcomes after cruciate-retaining TKA by affecting femorotibial articular contact kinematics.

\section{Methods}

Altogether, 20 knees in 10 patients who underwent posterior cruciate ligament-retaining TKA using the same size prosthesis for medial osteoarthritis were assessed preoperatively and 1 year postoperatively. PTS changes seen on lateral radiographs before and after TKA were calculated. Knees were placed in groups according to the PTS change at 1 year postoperatively (preoperative value - postoperative value). Group 1 had a $>3^{\circ}$ change and group 2 a $\leq 3^{\circ}$ change. Knee kinematics under the weight-bearing mid-flexion condition were compared between the two groups via two-dimensional/three-dimensional registration. Pain was measured using the visual analog scale, and knee function was based on the Western Ontario and McMaster universities (WOMAC) osteoarthritis index and Knee Society Score (KSS) questionnaire results.

\section{Results}

Group 2 experienced paradoxical anterior motion of the mediofemoral condyle postoperatively, whereas group 1 did not. Comparison of TKA results between the two groups showed a significant between-group difference in pain and knee function of the KSS and in the WOMAC osteoarthritis index score (P $\leq 0.05)$. Postoperative results were better in group 1 than in group 2.

\section{Conclusions}

Achieving a greater change in the posterior tibial slope apparently improves outcomes in patients undergoing posterior cruciate ligament-retaining TKA because it reduces the paradoxical medial femoral condylar movement.

\section{Introduction}

Outcomes after total knee arthroplasty (TKA) are affected by many factors, such as the preoperative state, type of prosthesis, surgeon's experience, surgical technique, rehabilitation, and a combination of any of the above factors [1].

Two-dimensional/three-dimensional (2D/3D) registration was used to evaluate knee kinematic patterns during the past two decades. Those studies indicated that the TKA outcomes were affected by knee kinematic patterns [2]. One study showed that A greater reduction in posterior tibial slope (PTS) after TKA compared with preoperative PTS reduces paradoxical medial femoral condylar movement. This may contribute to improved patient satisfaction after CR TKA [3]. We hypothesized that changes in PTS affect outcomes after cruciate-retaining TKA by affecting femorotibial articular contact kinematics.

\section{Materials And Methods}

Altogether, 20 knees of 10 patients (all women; average age 63.5 years, range 57-68 years at the time of surgery; average postoperative duration 12.4 months, range 12-13 months) underwent posterior cruciate-retaining (PCR)-TKA of the same size for medial osteoarthritis. They were assessed preoperatively and at 1 year after TKA. All 20 knees had varus deformity, which could flex $>90^{\circ}$ preoperatively.

Independent t-test and analysis of variance were used to compare postoperative results between the two groups. The level of significance was set at 0.05 .

Written informed consent was provided by all patients. The study design was approved by our institutional review board.

The PTS was determined by measuring the proximal tibial anatomical axis described by Utzschneider et al. in accordance with the subject's preoperative and postoperative lateral radiographic images [4]. The slope based on the configuration of the metal backing was measured (Figs. 1, 2).Knees were categorized into two groups $\left(>3^{\circ}\right.$, group 1,11 patients; $\leq 3^{\circ}$, group 2,9 patients) on the basis of the change in PTS, which was calculated by subtracting the postoperative value from the preoperative value (Table 1).

All PCR-TKAs were performed by the same senior surgeon using a knee PCR GEMINI prosthesis (Waldemar Link GmbH \& Co. KG, Hamburg, Germany). All TKAs were performed using a consistent, controllable, and reproducible technique. Proximal tibial osteotomy was performed with about $5^{\circ}$ of posterior inclination along the sagittal plane. The posterior cruciate ligament was retained when the anterior cruciate ligament was removed.

Postoperatively, each patient was required to accomplish a weight-bearing leg bend from full extension to $90^{\circ}$, which was monitored by a single fluoroscopic imaging system. The relative 3D position of the femoral and tibial prothesis was reproduced using a $2 \mathrm{D} / 3 \mathrm{D}$ registration method $[5,6]$. The $3 \mathrm{D}$ computerassisted design (CAD) models of the knee prostheses were imported into the software, where we created a complete match by manipulating the borders of the $\mathrm{CAD}$ and the corresponding prothesis on the lateral radiographic image (Fig. 3). The accuracy of the 2D/3D registration technique had been validated by prior experiments. The accuracy of the technique was within $0.5^{\circ}$ for rotations and $0.4 \mathrm{~mm}$ for displacement in the sagittal plane [3]. 
The bending angle was defined as the angle on the lateral radiographic image of the femur and the tibial component, and the contact point of the mediolateral femoral joint was defined as the nearest point between the CAD model femur and the tibial component [7]. In the tibial coordinate system, the $x$-axis was defined as the line connecting the medial point to the lateral point of the tibial surface, whereas the $y$-axis was defined as the axis that bisects and was perpendicular to the $\mathrm{x}$-axis in the transverse plane [8]. The anterior and posterior contact points of the $\mathrm{x}$-axis were designated as positive and negative, respectively.

All kinematics data were normally distributed. A repeated-measures analysis of variance and two independent samples $t$ tests were used to analyze the data. A value of $P<0.05$ was considered statistically significant. For a confidence level of $95 \%(a=0.05)$ and power $(1-\beta)$ of $80 \%$, a total sample size of 16 knees was needed.

\section{Results}

To compare knee kinematics between groups 1 and 2, we first looked at the femorotibial articular contact kinematics. In group 1 (Table 2 ), while bending the knee, the medial articular contact point continued to move backward. In contrast, in group 2 (Table 3), the medial articular contact point continued to move backward from $0^{\circ}$ to $20^{\circ}(\mathrm{P}<0.05)$. From $20^{\circ}$ to $50^{\circ}$, however, the point remained almost motionless $(\mathrm{P}>0.05)$. Then, from $50^{\circ}$ to $90^{\circ}$, it moved forward $(\mathrm{P}<$ 0.05). At $90^{\circ}$, the mean medial articular contact point in group 1 was significantly more forward than that of group $2(P=0.007)(F i g .4)$.

The lateral articular contact point in both groups moved back with different extents. The average lateral femorotibial articular contact point showed more significant posterior movement in group 1 than in group 2 during the entire bending process $(P<0.001)$ (Fig. 5).

A comparison of the TKAs between the two groups in the postoperative analysis shows that there was a significant difference between groups regarding pain and the knee function of the KSS and in the WOMAC osteoarthritis index score ( $(P \leq 0.05)$ (Table 4).

\section{Discussion}

The postoperative, comparative analysis of the TKAs in the two groups showed that the mean medial femorotibial articular contact point in group 1 was significantly more anterior than in group 2 . The medial femoral condyle of the knees in group 2 developed paradoxical anterior motion during the 1 -year recovery interval, whereas group 1 knees did not. There were also significant differences between the groups regarding pain and the functional Knee Society Score and the Western Ontario and McMaster universities osteoarthritis index scores $(P \leq 0.05)$. Thus, group 1 had better postoperative results than group 2 . A proper understanding of the reduced PTS in patients undergoing PCR-TKA, with the accompanying improved femorotibial articular contact kinematics, could contribute to better outcomes after PCR-TKA.

Previous studies focused on the influence of the tibial slope on extension and flexion gaps associated with TKA [9-11]. In recent years, some authors studied the influence of the tibial slope on knee kinematic patterns [12, 13]. Fujimoto stated that the mean medial femorotibial articular contact point in a large-PTS group was significantly more posterior than that in a small-PTS group, although there was no significant difference at the lateral femorotibial articular contact point during both weight bearing and non-weight bearing [13]. Previous studies have reported that changes in the knee kinematic patterns after TKA could affect outcomes, such as polyethylene wear and prosthetic loosening. Medial femoral condyle paradoxical anterior movement after PCR-TKA was also reported $[14,15]$. Another study noted a greater reduction in PTS after TKA compared with the preoperative PTS, thereby reducing the paradoxical mediofemoral condylar movement. The authors proposed a possible explanation for this mechanism [3].

The paradoxical movement after TKA has potential negative consequences, such as pathomechanics and decreased quadriceps efficiency, which could affect the outcome [16, 17].The biomechanics of a normal knee are changed by the paradoxical motion, which is non-physiological [18]. The dynamic balance of soft tissue-including the joint capsule, the lateral collateral ligament, and the patellofemoral joint-was disrupted. It causes discomfort during knee flexion, which in turn influences the postoperative recovery of knee function. Some believe that the paradoxical movement reduces the distance between the femorotibial articular contact point and the patellar tendon ligament tibial insertion during knee flexion, reduces the lever arm extensor mechanism, and leads to the requirement of greater quadriceps muscle force to extend the knee at higher flexion angles [19]. A large PTS could lead to the femorotibial articular contact point being in a posterior location, which would increase the efforts of the quadriceps of the lever arm and might cause movement inclination of the weight borne by the tibia [20,21]. This could lead to higher quadriceps efficiency, possibly resulting in discomfort during knee extension [22-24]. Furthermore, a more posterior femorotibial articular contact point than normal could cause the joint contact force to arrive at eccentric positions, resulting in abnormal compressive or tensile strain at the tibial bearing point $[18,25,26]$. It could therefore result in discomfort during weight bearing at mid-flexion.

There were both weaknesses and strengths in this study. One weakness was that the number of subjects was small. All patients underwent bilateral knee replacement, however, which reduced the influence of the small number of subjects. One strength was that all operations were completed by one senior surgeon using the same size PCR-TKA, applying a consistent, controllable technique. This reduced the random influences of the surgeon, prosthesis, and surgical technique.

In conclusion, a proper understanding of the reduced PTS in subjects undergoing PCR-TKA could improve the outcomes by affecting femorotibial articular contact kinematics. The reduced posterior tibial slope, however, could also lead to decreased knee flexion range of motion and increase the probability of direct impingement of the prosthesis on the posterior femur postoperatively [27-29]. Hence, the degree of reduction of the posterior tibial slope is a subject worthy of further study.

\section{Abbreviations}


PTS posterior tibial slope

WOMAC Western Ontario and McMaster universities

KSS Knee Society Score

2D/3D two-dimensional/three-dimensional

PCR posterior cruciate-retaining

CAD computer-assisted design

\section{Declarations}

\section{Acknowledgements}

We thank the Nancy Schatken BS, MT(ASCP), from Liwen Bianji, Edanz Group China (www.liwenbianji.cn/ac), to edit the English text of a draft of this manuscript to ensure the quality of the finished article.

\section{Ethics Approval and Consent to Participate}

All procedures performed in studies involving human participants were in accordance with the ethical standards of the institutional and/or national research committee and with the 1964 Helsinki declaration and its later amendments or comparable ethical standards. This article does not contain any studies with animals performed by any of the authors.

\section{Consent for publication}

Not applicable

\section{Availability of data and material}

The data is kept by the corresponding author and is available upon request.

\section{Competing interests}

On behalf of all authors, the corresponding author states that there is no conflict of interest.

\section{Authors' contributions}

Feng li and Xiqing Pan searched the literature and wrote the manuscript. All authors have read and approved the final manuscript.

\section{Funding}

This paper did not receive any funding.

\section{References}

1. Robertsson, O., et al., $₫ 2000 \bigotimes$ Patient satisfaction after knee arthroplasty: a report on 27,372 knees operated on between 1981 and 1995 in Sweden. Acta Orthop Scand. 71(3): p. 262-7.

2. Victor, J. and J. Bellemans, $82006 \square$ Physiologic kinematics as a concept for better flexion in TKA. Clin Orthop Relat Res. 452: p. 53-8.

3. Pan, X.Q., et al., $₫ 2017 \rrbracket$ Effect of tibial slope changes on femorotibial contact kinematics after cruciate-retaining total knee arthroplasty. Knee Surg Sports Traumatol Arthrosc.25邓p.3549-55

4. Utzschneider, S., et al., $\varangle 2011 \rrbracket$ Development and validation of a new method for the radiologic measurement of the tibial slope. Knee Surg Sports Traumatol Arthrosc. 19(10): p. 1643-8.

5. Dennis, D.A., R.D. Komistek, and M.R. Mahfouz, $₫ 2003 \rrbracket$ In vivo fluoroscopic analysis of fixed-bearing total knee replacements. Clin Orthop Relat Res. (410): p. 114-30.

6. Hoff, W.A., et al., $₫ 1998 \rrbracket$ Three-dimensional determination of femoral-tibial contact positions under in vivo conditions using fluoroscopy. Clin Biomech (Bristol, Avon). 13(7): p. 455-472.

7. Shimizu, N., et al., $₫ 2013 \bigotimes$ Posterior sliding of the femur during stair ascending and descending in a high-flex posterior stabilized total knee arthroplasty. $\mathrm{J}$ Arthroplasty. 28(10): p. 1707-11.

8. Li, G., T.H. Wuerz, and L.E. DeFrate, $₫ 2004 \rrbracket$ Feasibility of using orthogonal fluoroscopic images to measure in vivo joint kinematics. J Biomech Eng. 126(2): p. 314-8.

9. Mihalko, W.M. and K.A. Krackow, $₫ 2006 \bigotimes$ Flexion and extension gap balancing in revision total knee arthroplasty. Clin Orthop Relat Res. 446: p. 121-6. 
10. Minoda, Y., et al., $\llbracket 2007 \rrbracket$ Flexion gap preparation opens the extension gap in posterior cruciate ligament-retaining TKA. Knee Surg Sports Traumatol Arthrosc. 15(11): p. 1321-5.

11. Krackow, K.A. and W.M. Mihalko, $₫ 2001 \rrbracket$ The effects of severe femoral bone loss on the flexion extension joint space in revision total knee arthroplasty: a cadaveric analysis and clinical consequences. Orthopedics. 24(2): p. 121-6.

12. Matsuzaki, $T$., et al., $₫ 2013 \rrbracket$ Kinematic factors affecting postoperative knee flexion after cruciate-retaining total knee arthroplasty. Int Orthop. 37(5): p. 8038.

13. Fujimoto, E., et al., $₫ 2014 \llbracket$ Significant effect of the posterior tibial slope on the weight-bearing, midflexion in vivo kinematics after cruciate-retaining total knee arthroplasty. J Arthroplasty. 29(12): p. 2324-30.

14. Sharkey, P.F., et al., $₫ 2002 \bigotimes$ Insall Award paper. Why are total knee arthroplasties failing today? Clin Orthop Relat Res. (404): p. 7-13.

15. Harman, M.K., S.A. Banks, and W.A. Hodge, $₫ 2001 \rrbracket$ Polyethylene damage and knee kinematics after total knee arthroplasty. Clin Orthop Relat Res. (392): p. 383-93.

16. Dennis, D.A., et al., $₫ 2007 \rrbracket$ Factors affecting flexion after total knee arthroplasty. Clin Orthop Relat Res. 464: p. 53-60.

17. Dennis, D.A., et al., $1996 \rrbracket$ In vivo knee kinematics derived using an inverse perspective technique. Clin Orthop Relat Res. (331): p. 107-17.

18. Jojima, H., L.A. Whiteside, and K. Ogata, $₫ 2004 \llbracket$ Effect of tibial slope or posterior cruciate ligament release on knee kinematics. Clin Orthop Relat Res. (426): p. 194-8.

19. Ostermeier, S., et al., $₫ 2006 \bigotimes$ In vitro investigation of the influence of tibial slope on quadriceps extension force after total knee arthroplasty. Knee Surg Sports Traumatol Arthrosc. 14(10): p. 934-9.

20. Giffin, J.R., et al., $₫ 2004 \rrbracket$ Effects of increasing tibial slope on the biomechanics of the knee. Am J Sports Med. 32(2): p. $376-82$.

21. Hernigou, P. and G. Deschamps, $₫ 2004 \rrbracket$ Posterior slope of the tibial implant and the outcome of unicompartmental knee arthroplasty. J Bone Joint Surg Am. 86-A(3): p. 506-11.

22. Garg, A. and P.S. Walker, $11990 \otimes$ Prediction of total knee motion using a three-dimensional computer-graphics model. J Biomech. 23(1): p. 45-58.

23. Godest, A.C., et al., $₫ 2000 \rrbracket$ A computational model for the prediction of total knee replacement kinematics in the sagittal plane. J Biomech. 33(4): p. 43542.

24. Walker, P.S. and S. Sathasivam, $11999 \bigotimes$ The design of guide surfaces for fixed-bearing and mobile-bearing knee replacements. J Biomech. 32(1): p. 27-34.

25. Piazza, S.J., et al., $\$ 1998 \rrbracket$ Posterior tilting of the tibial component decreases femoral rollback in posterior-substituting knee replacement: a computer simulation study. J Orthop Res. 16(2): p. 264-70.

26. Catani, F., et al., $₫ 2004 \bigotimes$ The stability of the cemented tibial component of total knee arthroplasty: posterior cruciate-retaining versus posterior-stabilized design. J Arthroplasty. 19(6): p. 775-82.

27. Bellemans, J., et al., $₫ 2002 \rrbracket$ Fluoroscopic analysis of the kinematics of deep flexion in total knee arthroplasty. Influence of posterior condylar offset. J Bone Joint Surg Br. 84(1): p. 50-3.

28. Massin, P. and A. Gournay, \2006囚 Optimization of the posterior condylar offset, tibial slope, and condylar roll-back in total knee arthroplasty. J Arthroplasty. 21(6): p. 889-96.

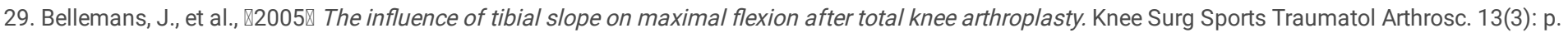
193-6.

\section{Tables}

Table1. PTS $\left({ }^{\circ}\right)$, preoperative value and postoperative value from

\begin{tabular}{|lllll|}
\hline & Group 1 & \multicolumn{3}{c|}{ Group 2 } \\
\cline { 2 - 5 } & preoperative & postoperative & preoperative & postoperative \\
\hline 1 & 12.2 & 4.8 & 9.6 & 8.2 \\
\hline 2 & 11.1 & 4.8 & 6.7 & 8.1 \\
\hline 3 & 12.0 & 6.0 & 3.2 & 10.2 \\
\hline 4 & 11.0 & 7.9 & 7.5 & 4.7 \\
\hline 5 & 12.1 & 4.3 & 12.1 & 12.2 \\
\hline 6 & 11.1 & 7.4 & 5.9 & 8.4 \\
\hline 7 & 17.7 & 13.6 & 8.6 & 6.8 \\
\hline 8 & 14.8 & 10.7 & 9.5 & 8.1 \\
\hline 9 & 14.5 & 9.8 & 13.6 & 14.1 \\
\hline 10 & 14.9 & 8.5 & & \\
\hline 11 & 8.8 & 3.2 & & \\
\hline
\end{tabular}


Table2. Group 1 AP Translation (mm), Femorotibial Articular Contact Point

\begin{tabular}{|c|c|c|c|c|c|c|c|c|c|c|c|c|c|c|c|c|c|c|c|}
\hline \multirow{2}{*}{$\begin{array}{l}\text { Subject } \\
\text { No }\end{array}$} & \multicolumn{10}{|l|}{ Med } & \multicolumn{9}{|l|}{ Lat } \\
\hline & $0^{\circ}$ & $10^{\circ}$ & $20^{\circ}$ & $30^{\circ}$ & $40^{\circ}$ & $50^{\circ}$ & $60^{\circ}$ & $70^{\circ}$ & $80^{\circ}$ & $90^{\circ}$ & $0^{\circ}$ & $10^{\circ}$ & $20^{\circ}$ & $30^{\circ}$ & $40^{\circ}$ & $50^{\circ}$ & $60^{\circ}$ & $70^{\circ}$ & $80^{\circ}$ \\
\hline 1 & 0.1 & -1.3 & -1.8 & -2.9 & -3.3 & -2.5 & -3.7 & -5.4 & -6.0 & -6.2 & -0.1 & -2.4 & -3.6 & -3.9 & -6.1 & -3.2 & -7.5 & -9.4 & -9.6 \\
\hline 2 & 1.9 & 1.2 & 0.8 & -0.5 & -1.2 & -1.5 & -3.3 & -4.1 & -5.5 & -5.9 & -1.9 & -2.6 & -3.3 & -3.3 & -3.8 & -4.5 & -6.8 & -7.2 & -8.9 \\
\hline 3 & -3.1 & -4.2 & -5.5 & -7.3 & -7.5 & -7.8 & -8.1 & -9.6 & -10.2 & -11.4 & 0.5 & -0.8 & -1.2 & -2.6 & -3.7 & -4.2 & -5.7 & -6.6 & -9.2 \\
\hline 4 & -0.5 & -0.8 & -0.9 & -1.5 & -2.1 & -2.2 & -2.4 & -2.4 & -2.6 & -2.6 & -0.2 & -1.1 & -2.3 & -3.1 & -3.6 & -3.9 & -4.5 & -6.0 & -7.4 \\
\hline 5 & -0.7 & -1.1 & -1.6 & -2.8 & -3.1 & -3.2 & -3.3 & -4.2 & -5.4 & -5.9 & -1.8 & -2.2 & -2.5 & -3.7 & -5.5 & -6.9 & -7.2 & -8.2 & -10.5 \\
\hline 6 & 1.0 & 0.7 & 0.6 & 0.6 & 0.4 & 0.2 & 0.2 & -0.2 & -0.4 & -0.8 & -0.5 & -1.2 & -1.5 & -2.8 & -3.6 & -3.8 & -4.9 & -5.5 & -7.5 \\
\hline 7 & -0.9 & -1.0 & -1.2 & -1.3 & -1.7 & -2.1 & -1.9 & -1.7 & -0.9 & -0.7 & -0.5 & -1.5 & -2.9 & -4.1 & -4.7 & -5.2 & -7.3 & -7.8 & -9.9 \\
\hline 8 & 0.1 & -0.3 & -0.5 & -0.9 & -1.0 & -1.2 & -1.6 & -1.7 & -1.7 & -1.9 & -1.6 & -2.0 & -3.4 & -3.9 & -4.9 & -5.2 & -6.7 & -7.1 & -9.2 \\
\hline 9 & 1.7 & 1.2 & 0.9 & 0.3 & -0.1 & -0.2 & -0.5 & -0.9 & -0.9 & -1.1 & -1.9 & -2.8 & -3.9 & -4.2 & -5.8 & -6.8 & -7.5 & -7.7 & -10.2 \\
\hline 10 & -0.9 & -1.5 & -1.9 & -2.6 & -2.8 & -3.5 & -3.5 & -3.8 & -3.8 & -4.1 & -3.1 & -4.2 & -4.8 & -6.7 & -7.2 & -7.5 & -8.9 & -9.6 & -10.8 \\
\hline 11 & -0.2 & -0.6 & -0.8 & -1.5 & -2.0 & -2.1 & -2.4 & -2.6 & -2.8 & -3.1 & -0.8 & -1.9 & -2.7 & -3.5 & -4.0 & -4.6 & -6.2 & -7.3 & -9.8 \\
\hline Mean & -0.1 & -0.7 & -1.1 & -1.9 & -2.2 & -2.4 & -2.8 & -3.3 & -3.7 & -4.0 & -1.1 & -2.1 & -2.9 & -3.8 & -4.8 & -5.1 & -6.7 & -7.5 & -9.4 \\
\hline SD & 1.4 & 1.5 & 1.8 & 2.1 & 2.1 & 2.1 & 2.2 & 2.6 & 2.9 & 3.2 & 1.1 & 1.0 & 1.0 & 1.1 & 1.2 & 1.4 & 1.3 & 1.3 & 1.1 \\
\hline
\end{tabular}

Table 3 Group 2 AP Translation (mm), Femorotibial Articular Contact Point

\begin{tabular}{|c|c|c|c|c|c|c|c|c|c|c|c|c|c|c|c|c|c|c|c|}
\hline \multirow{2}{*}{$\begin{array}{l}\text { Subject } \\
\text { No. }\end{array}$} & \multicolumn{10}{|c|}{ Med } & \multicolumn{9}{|l|}{ Lat } \\
\hline & $0^{\circ}$ & $10^{\circ}$ & $20^{\circ}$ & $30^{\circ}$ & $40^{\circ}$ & $50^{\circ}$ & $60^{\circ}$ & $70^{\circ}$ & $80^{\circ}$ & $90^{\circ}$ & $0^{\circ}$ & $10^{\circ}$ & $20^{\circ}$ & $30^{\circ}$ & $40^{\circ}$ & $50^{\circ}$ & $60^{\circ}$ & $70^{\circ}$ & $80^{\circ}$ \\
\hline 1 & 1.9 & 1.8 & 0.3 & -0.1 & -0.9 & -0.8 & 0.4 & 0.8 & 1.0 & 1.5 & -2.9 & -3.5 & -3.6 & -4.8 & -4.9 & -6.8 & -5.1 & -7.2 & -8.1 \\
\hline 2 & 1.9 & -1.2 & -6.1 & -5.5 & -5.6 & -3.9 & -3.5 & -2 & -1.8 & -0.9 & -2.1 & -2.6 & -2.9 & -4.8 & -5.2 & -5.5 & -6.5 & -6.7 & -7.2 \\
\hline 3 & 2.7 & 1.8 & -1.5 & -1.9 & 0.1 & 0.2 & 0.8 & 1.2 & 1.6 & 2.2 & -0.1 & -1.2 & -1.5 & -2.6 & -2.8 & -3.2 & -4.1 & -4.4 & -4.5 \\
\hline 4 & -1.0 & -1.1 & -1.1 & -1.2 & -1.3 & -1.5 & -1.5 & -1.4 & -1.4 & -1.2 & -3.2 & -3.5 & -4.8 & -5.0 & -5.2 & -6.3 & -6.5 & -7.6 & -10.2 \\
\hline 5 & -2.1 & -2.6 & -3.2 & -3.5 & -4.8 & -5.0 & -4.2 & -4.1 & -3.4 & -3.1 & -6.0 & -6.3 & -6.7 & -6.9 & -7.8 & -8.5 & -8.8 & -9.6 & -10.1 \\
\hline 6 & -0.3 & -1.2 & -2.1 & -2.4 & -2.3 & -2.1 & -1.6 & -1.1 & -0.8 & -0.5 & -1.7 & -2.4 & -2.8 & -3.9 & -4.1 & -4.5 & -4.8 & -5.5 & -5.8 \\
\hline 7 & 0.9 & 0.2 & -1.1 & -2.9 & -3.2 & -3.4 & -2.5 & -1.0 & -0.2 & 0.2 & -3.9 & -4.6 & -5.2 & -6.3 & -6.6 & -7.1 & -8.4 & -9.6 & -9.8 \\
\hline 8 & -2.8 & -3.1 & -3.2 & -3.6 & -3.9 & -4.1 & -3.2 & -3.0 & -2.3 & -2.1 & -2.2 & -3.1 & -3.4 & -4.8 & -5.3 & -6.6 & -7.5 & -7.6 & -8.5 \\
\hline 9 & 0.3 & -0.5 & -1.0 & -2.3 & -2.4 & -2.6 & -2.5 & -1.8 & -.0 .9 & -0.6 & -2.6 & -3.0 & -3.5 & -4.1 & -4.3 & -5.0 & -6.1 & -6.9 & -7.2 \\
\hline Mean & 0.2 & -0.7 & -2.3 & -2.6 & -2.7 & -2.6 & -1.9 & -1.3 & -0.9 & -0.5 & -2.8 & -3.4 & -3.9 & -4.9 & -5.2 & -6.1 & -6.5 & -7.3 & -8.0 \\
\hline SD & 2.0 & 1.8 & 1.9 & 1.6 & 2.0 & 1.8 & 1.8 & 1.8 & 1.7 & 1.8 & 1.7 & 1.5 & 1.6 & 1.3 & 1.5 & 1.6 & 1.7 & 1.8 & 2.1 \\
\hline
\end{tabular}

Table 4. Comparison of test parameters between groups before and after operation

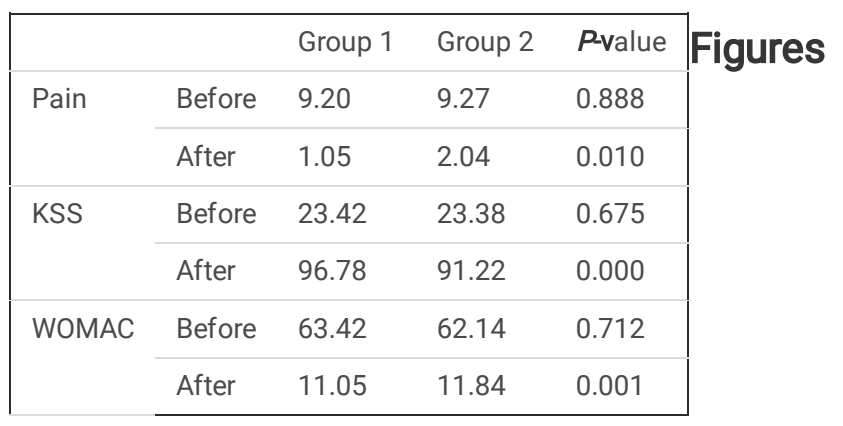




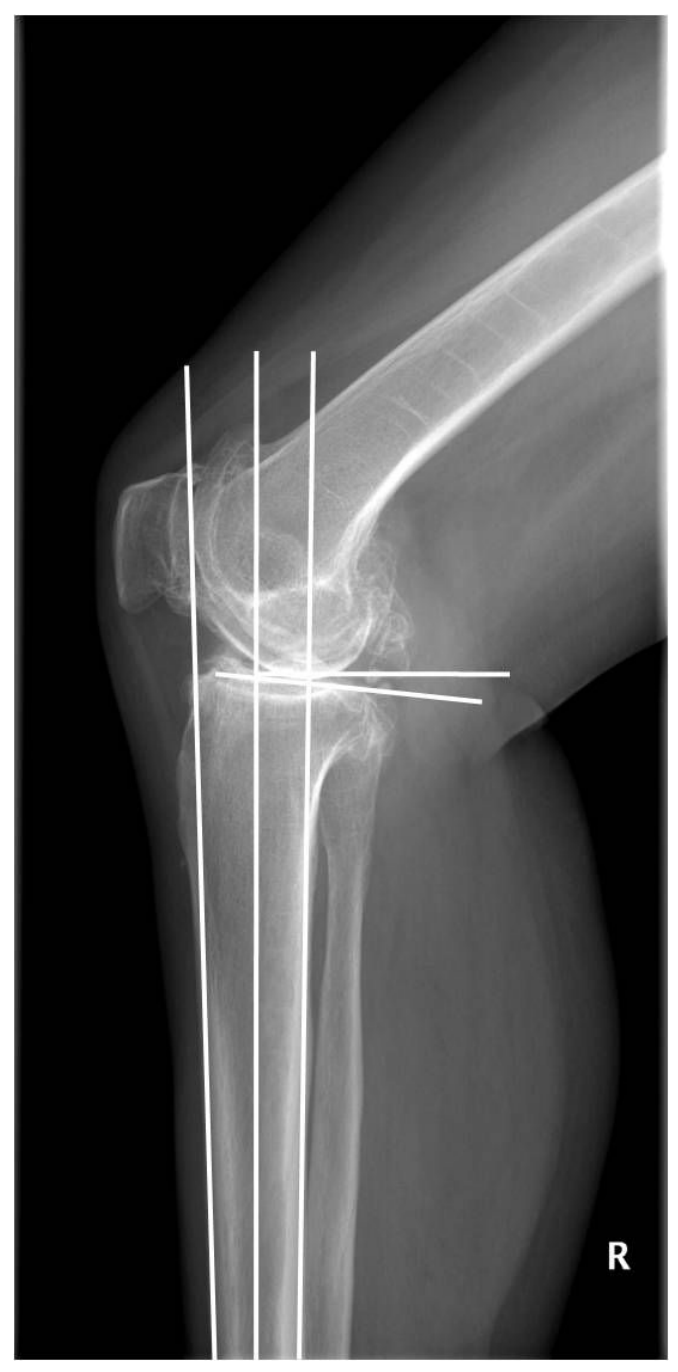

\section{Figure 1}

The PTS was determined by measuring the proximal tibial anatomical axis described by Utzschneider et al. in accordance with the subject's preoperative and postoperative lateral radiographic images [4]. The slope based on the configuration of the metal backing was measured 


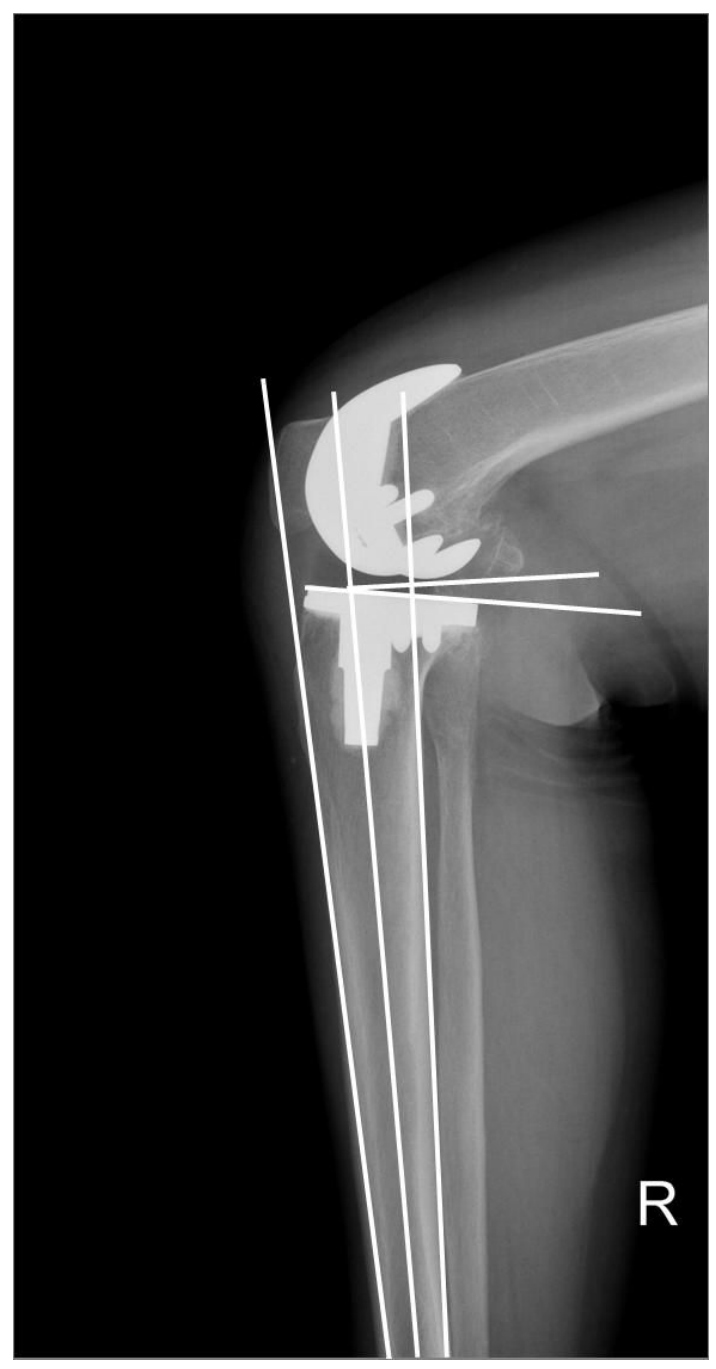

\section{Figure 2}

The PTS was determined by measuring the proximal tibial anatomical axis described by Utzschneider et al. in accordance with the subject's preoperative and postoperative lateral radiographic images [4]. The slope based on the configuration of the metal backing was measured

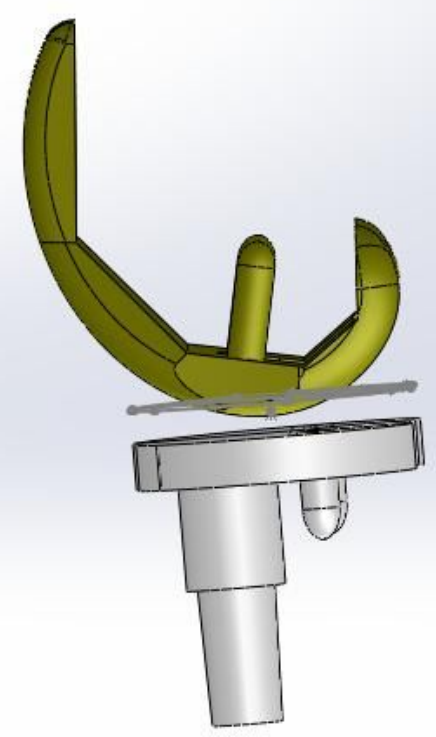


The 3D computer-assisted design (CAD) models of the knee prostheses were imported into the software, where we created a complete match by manipulating the borders of the $\mathrm{CAD}$ and the corresponding prothesis on the lateral radiographic image

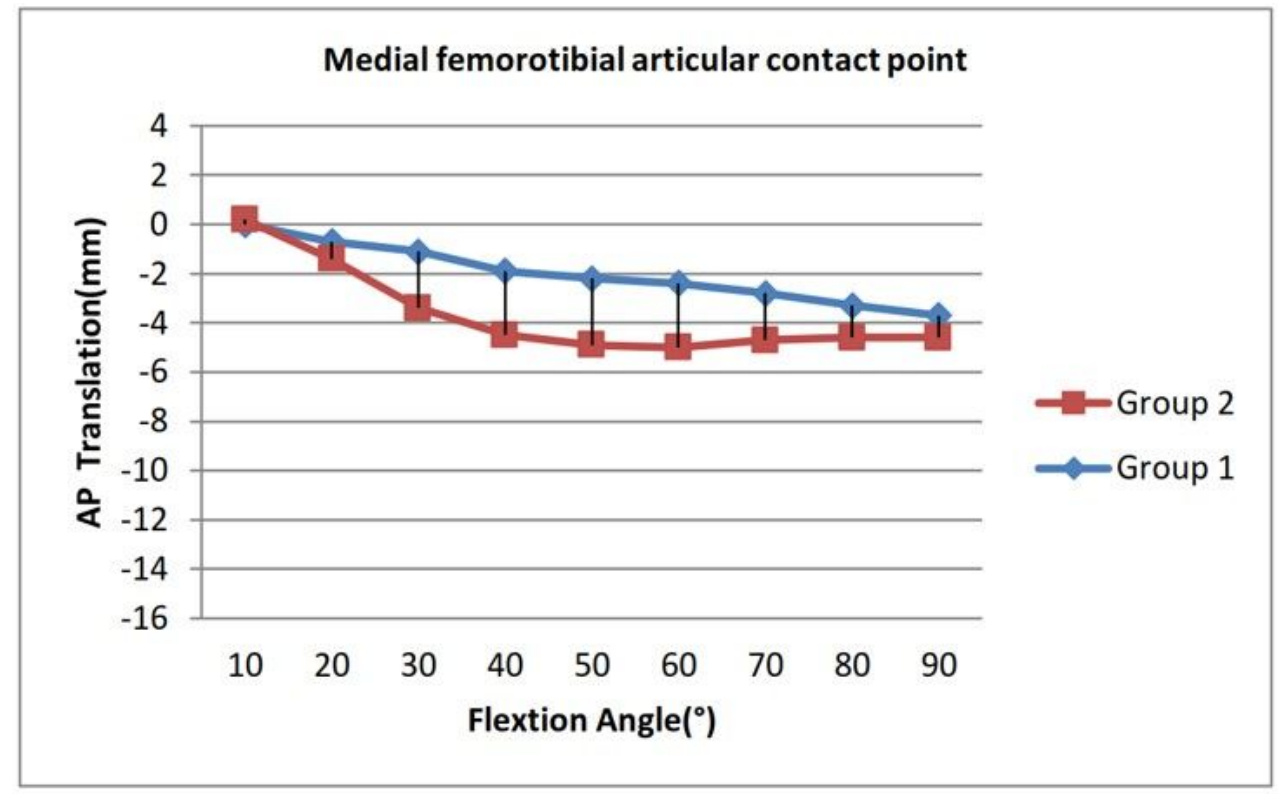

\section{Figure 4}

compare knee kinematics between groups 1 and 2, we first looked at the femorotibial articular contact kinematics. In group 1 (Table 2 ), while bending the knee, the medial articular contact point continued to move backward. In contrast, in group 2 (Table 3), the medial articular contact point continued to move backward from $0^{\circ}$ to $20^{\circ}(\mathrm{P}<0.05)$. From $20^{\circ}$ to $50^{\circ}$, however, the point remained almost motionless $(\mathrm{P}>0.05)$. Then, from $50^{\circ}$ to $90^{\circ}$, it moved forward $(\mathrm{P}<$ 0.05). At $90^{\circ}$, the mean medial articular contact point in group 1 was significantly more forward than that of group $2(P=0.007)$

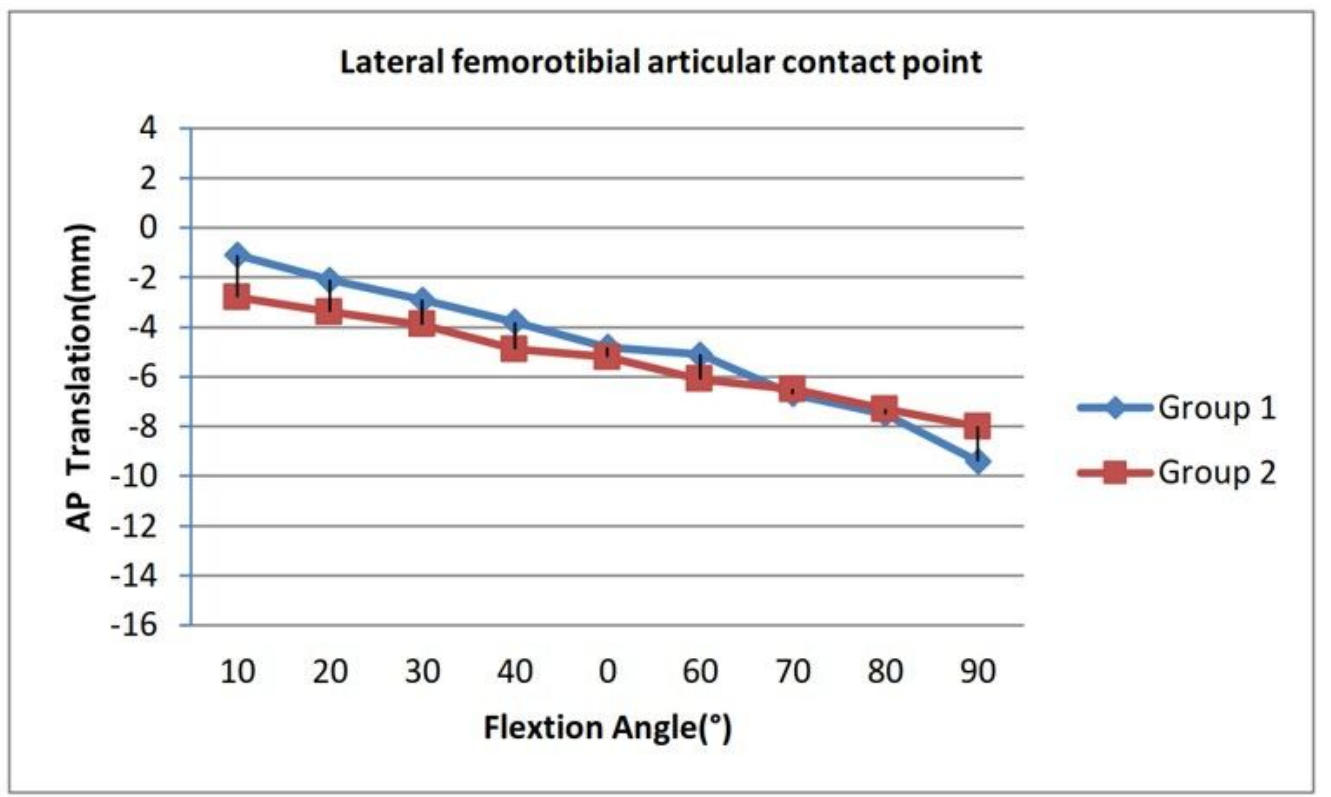

Figure 5

The lateral articular contact point in both groups moved back with different extents. The average lateral femorotibial articular contact point showed more significant posterior movement in group 1 than in group 2 during the entire bending process $(P<0.001)$ 